\title{
Olmesartan Prevented Intra-articular Inflammation Induced by Zymosan in Rats
}

\author{
Gerlane Coelho Bernardo Guerra, ${ }^{a}$ Marília Stefani Souza de Menezes, ${ }^{b}$ \\ Aurigena Antunes de Araújo, ${ }^{c}$ Raimundo Fernandes de Araújo Júnior, ${ }^{d}$ and \\ Caroline Addison Carvalho Xavier de Medeiros*,e \\ ${ }^{a}$ Post Graduation Program in Biological Sciences/Federal University of Rio Grande do Norte, Department \\ Biophysical and Pharmacology, Federal University of Rio Grande do Norte; Av. Senador Salgado Filho, S/N, \\ Lagoa Nova, Natal, Rio Grande do Norte 59072-970, Brazil: ${ }^{b}$ Program Post-Graduation in Health and Society/ \\ State University of Rio Grande do Norte; Mossoró, RN, Cep 59078-970, Brazil: ' Post Graduation Program Public \\ Health/Post Graduation Program in Pharmaceutical Science/Department of Biophysics and Pharmacology, Federal \\ University of Rio Grande do Norte; Natal, Rio Grande do Norte 59072-970, Brazil: ${ }^{d}$ Post Graduation Program \\ in Functional and Structural Biology/Post Graduation Program Health Science/Department of Morphology, \\ Federal University of Rio Grande do Norte; Natal, Rio Grande do Norte 59072-970, Brazil: and ${ }^{e}$ Department of \\ Biophysics and Pharmacology, Post Graduation Program in Biotechnology RENORBIO, Post Graduation Program \\ in Biological Sciences/Federal University of Rio Grande do Norte, Post Graduation Program in Health and Society/ \\ State University of Rio Grande do Norte; Natal, Rio Grande do Norte 59072-970, Brazil. \\ Received March 29, 2016; accepted July 26, 2016
}

The objective of this study was to study the effect of olmesartan medoxomil (OLM), an antihypertensive drug, on intra-articular inflammation induced by zymosan $(\mathrm{Zy})$ in Wistar rats. Intra-articular inflammation was induced in the right knees of rats by $1 \mathrm{mg} \mathrm{Zy}$ dissolved in saline. The animals were divided into the following groups: saline only (oral saline and intra-articular saline); Zy only (intra-articular Zy and oral saline), and intra-articular Zy and oral OLM (5, 15, or $30 \mathrm{mg} / \mathrm{kg}$ ) or diclofenac sodium (SD; $100 \mathrm{mg}$ / $\mathrm{kg}$ ). Twenty-four hours after $\mathrm{Zy}$ injection, synovial fluid was collected for total leukocyte counts, blood was collected for biochemical measurements, and synovial tissue was collected for histopathology, immunohistochemistry, immunofluorescence and myeloperoxidase (MPO), malonaldehyde (MDA), and non-protein sulphydryl (NPSH) assays. OLM doses of 15 and $30 \mathrm{mg} / \mathrm{kg}$ had protective effects, as evidenced by improved histopathological parameters of synovium, reduced total leukocyte counts, reduced MPO and MDA levels, and increased NPSH group levels compared with the Zy group. OLM reduced immunostaining for cyclooxygenase 2, tumour necrosis factor and interleukin 17 and increased immunostaining for superoxide dismutase and glutathione peroxidase. SD produced similar results. The drugs studied caused no change in biochemical parameters of the animals. OLM showed protective effects in this model of Zy-induced intra-articular inflammation.

Key words olmesartan; zymosan; intra-articular inflammation

Rheumatoid arthritis (RA) is a chronic inflammatory synovitis accompanied by the destruction of articular cartilage and bone. Articular inflammation is a limiting step in disease development and for structural damage. ${ }^{1)}$ Zymosan (Zy), a derivative of Saccharomyces cerevisiae yeast, induces joint inflammation mediated by toll-like receptor 2 (TLR2). ${ }^{2} \mathrm{Zy}$ induced articular inflammation is an experimental model of RA described in the literature, and a useful tool for the study of substances' anti-inflammatory activities. ${ }^{3)}$

The acute phase of Zy-induced RA is characterised by increased vascular permeability, oedema, and inflammatory infiltration, which peaks in the 6th hour with a polymorphonuclear predominance. Increasing mononuclear cell infiltration and fibroblast activation occurs, similar to that in chronic rheumatoid synovitis, in which the synovial tissue grows to cover the cartilage (rheumatoid pannus). ${ }^{3)}$

The pathogenesis of RA is complex and incompletely understood. Oxidative stress plays an important role. ${ }^{4)}$ Reactive oxygen species (ROS) are produced by cellular processes and oxidative phosphorylation. An excess of ROS leads to lipid peroxidation of the cell membrane and abuse of tissue proteins, carbohydrates, and DNA. Patients with RA have high oxidative stress levels and higher malonaldehyde (MDA) levels than do normal patients. ${ }^{5)}$ Data suggest that an antioxidant diet can reduce RA incidence. ${ }^{6}$

RA is considered to be a systemic disease that primarily affects the joints, but inflammatory mediators involved in the pathogenesis of the disease can affect other organs, such as the heart. Patients with RA have higher incidence of and mortality from cardiovascular disease (CVD), including myocardial infarction, cerebrovascular events, and heart failure. Proinflammatory cytokines, immune complexes, acute phase reactants, and modified lipid particles increase endothelial cell activation and potentially atherosclerotic plaque instability. ${ }^{7)}$

RA has been treated with anti-inflammatory, ${ }^{8)}$ immunosuppressive, ${ }^{9)}$ and biologic agents such as infliximab and interleukin-35. ${ }^{10,11)}$ Clinical results remain limited, and research on new therapies is needed.

Olmesartan medoxomil (OLM) is an angiotensin II type 1 receptor blocker (ARB), used clinically for the treatment of primary essential hypertension. This drug has an anti-inflammatory effect on the atherosclerotic process and improves cardiovascular dysfunction. ${ }^{12)}$ The anti-inflammatory activities of other ARBs have been described in the literature. Valsar- 
tan had a protective effect in an experimental model of acute pancreatitis. $^{13)}$ Irbesartan attenuated tumor necrosis factor (TNF)- $\alpha$-induced intercellular adhesion molecule (ICAM)-1, vascular cell adhesion molecule (VCAM)-1, and E-selectin expression levels in an in vitro model. ${ }^{14)}$ Losartan attenuated lipopolysaccharide-induced lung injury. ${ }^{15)}$ Telmisartan reduced the inflammatory response in an experimental model of periodontitis, ${ }^{16)}$ and azilsartan prevented oral mucositis induced by 5-fluorouracil. ${ }^{17)}$ In the renin-angiotensin system, angiotensin II participates in the pathogenesis of atherosclerosis through effects on endothelial function, inflammation, ROS production, and proliferation/migration of vascular cells. ${ }^{18)}$ In this study, we investigated the effect of OLM on Zy-induced acute articular inflammation in a rat model.

\section{MATERIALS AND METHODS}

Animals and Zymosan (Zy)-Induced Articular Inflammation Model Male Wistar rats weighing 180-220g were used in the experimental model. The animals were housed in polypropylene cages ( 2 animals per cage), with food and water provided ad libitum, in a temperature-controlled room $\left(22 \pm 1^{\circ} \mathrm{C}\right)$ with a $12 / 12$-h light/dark cycle. The Committee on Ethics in Animal Experiments of the Federal University of Rio Grande do Norte approved the study protocol (permit number 016-2013).

For the experimental model of Zy-induced joint inflammation, rats were anesthetised with ketamine $(70 \mathrm{mg} / \mathrm{kg})$ and xylazine $(10 \mathrm{mg} / \mathrm{kg})$, followed by intra-articular injection into the right knee of a $0.05-\mathrm{mL}$ solution of $1 \mathrm{mg} \mathrm{Zy}$ dissolved in saline. The animals were divided into experimental groups of five animals each $(n=5)$. Normal rats received intra-articular and oral (via gavage) saline. Animals in the Zy group received intra-articular Zy and oral saline. The treated groups received intra-articular Zy and oral OLM at different doses (5, 15, or $30 \mathrm{mg} / \mathrm{kg})^{19)}$ or oral diclofenac sodium (SD) at a dose of $100 \mathrm{mg} / \mathrm{kg} .{ }^{20)}$ OLM and SD were administered orally $30 \mathrm{~min}$ before $\mathrm{Zy}$ injection. The saline was used to dissolve olmesartan and diclofenac sodium. The anti-inflammatory effect of OLM was compared to that of SD, a nonsteroidal anti-inflammatory drug used in clinical practice.

The rats were euthanized with anaesthetic thiopental $(80 \mathrm{mg} / \mathrm{kg}) 24 \mathrm{~h}$ after induction of inflammation. Synovial fluid was used for total leukocyte counts, and synovial membranes were collected for histopathology and immunohistochemistry. The synovium was frozen at $-80^{\circ} \mathrm{C}$ and processed for the analysis of myeloperoxidase (MPO) activity, non-protein sulphydryl (NPSH) groups, and MDA level. Blood collection was performed for the analysis of serum urea, creatinine, alanine aminotransferase (ALT), and aspartate aminotransferase (AST) levels.

Synovial Fluid Collection and Total Leukocyte Count Synovial fluid collection was performed with the administration of two intra-articular injections of sodium phosphate buffer $(0.15 \mathrm{M}, \mathrm{pH} 7.4)$ and ethylenediaminetetraacetic acid (EDTA) $(0.01 \mathrm{M})$, followed by aspiration. Synovial fluid $(20 \mu \mathrm{L})$ was diluted with $380 \mu \mathrm{L}$ Turk solution for leukocyte counts. ${ }^{21)}$

Histopathology The synovium was fixed in $10 \%$ buffered formaldehyde for $24 \mathrm{~h}$ and processed for staining with hematoxylin and eosin according to a previously described technique. ${ }^{22)}$ Microscopic analysis was performed to evaluate aspects such as inflammatory cell infiltration, fibrosis, and granuloma.

MPO Activity MPO activity in synovia was assessed using a modified version of the method described by Bradley et al. $^{23)}$ Samples were incubated in a $0.5 \%$ hexadecyltrimethylammonium bromide solution, homogenised, and centrifuged. MPO activity was determined by a colorimetric method, with absorbance at $460 \mathrm{~nm}$.

Glutathione (GSH) Assay GSH concentrations were determined by testing for NPSH groups. ${ }^{24)}$ Samples of synovium were triturated with $0.02 \mathrm{M}$ EDTA, and $50 \%$ trichloroacetic acid was used to precipitate the protein. Absorbance was determined at $412 \mathrm{~nm}$ immediately after the addition of 5,5dithiobis(2-nitrobenzoic acid).

Malondialdehyde Assay We use a previously described method with a high degree of specificity to detect MDA. ${ }^{25)}$ Samples of synovium were homogenised with $20 \mathrm{~mm}$ Tris- $\mathrm{HCl}$ and centrifuged. MDA reacts with the chromogen 1-methyl2-phenylindole, with maximum absorbance at $586 \mathrm{~nm}$.

Immunohistochemistry for the Analysis of Cyclooxygenase-2 (COX-2), Superoxide Dismutase (SOD), Glutathione Peroxidise (GSH-Px), TNF and Interleukin 17 (IL 17) Synovial tissue was analysed immunohistochemically to detect COX-2, SOD, GSH-Px, TNF and IL 17. The biotin-streptavidin-peroxidase technique as used. ${ }^{26}$ ) The samples were incubated with primary antibody, and then with biotinylated secondary antibody. Peroxidase-streptavidin binds to the biotin

(a)

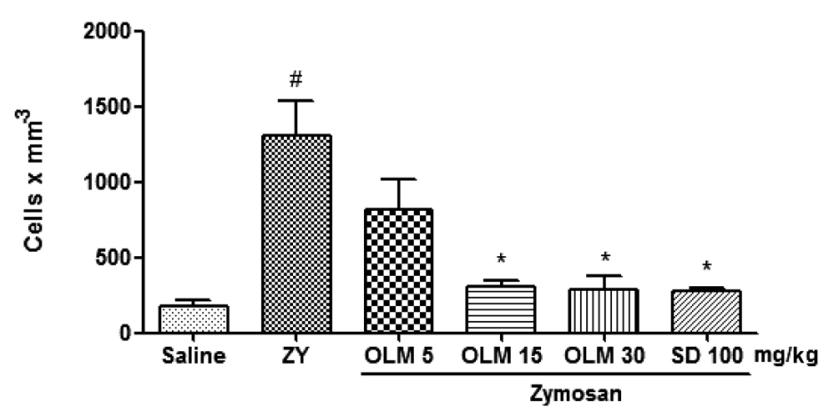

(b)

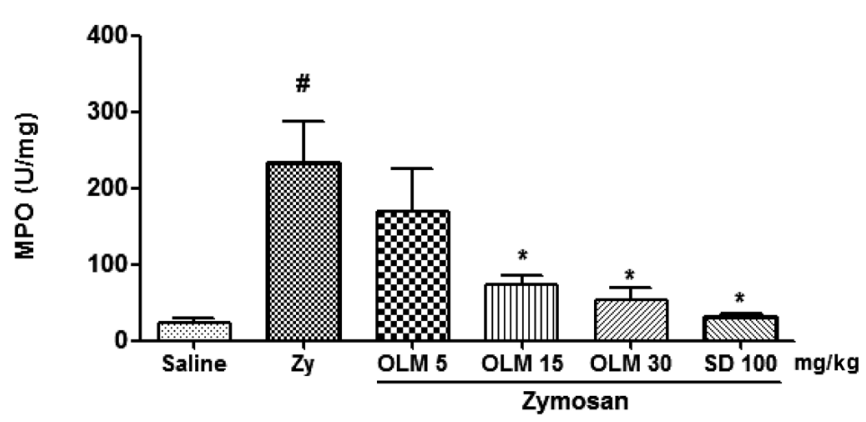

Fig. 1. Effects of Olmesartan on Intra-articular Inflammation Induced by Zymosan

(a) Total leukocyte counts in synovial fluid. (b) Myeloperoxidase activity in synovia. Rats received intra-articular saline alone (saline), intra-articular zymosan alone (Zy), and intra-articular zymosan and olmesartan (OLM; 5, 15, or 30 mg/ $\mathrm{kg}$ ) or diclofenac sodium (SD; $100 \mathrm{mg} / \mathrm{kg}$ ). OLM and SD were administered orally 30 min before zymosan injection. Results are presented as the mean \pm S.E.M. $(n=5)$. ${ }^{\#} p<0.05$ vs. saline, ${ }^{*} p<0.05$ vs. Zy (ANOVA with Tukey's post-test). 
molecule attached to the secondary antibody. The chromogen (DAB) reacts with the peroxidase linked to streptavidin, emitting colour.

Immunofluorescence for the Analysis of TNF and IL 17 Synovial tissue was analysed by immunofluorescence to detect TNF and IL 17, according to a previously described tech- nique. ${ }^{27)}$ The samples (five samples per group) were incubated overnight with primary antibody, then washed three times in PBS $/ 0.2 \%$ Triton X-100 for $5 \mathrm{~min}$ and incubated with Alexa Fluor 488-conjugated secondary antibody and 4'-6-diamidino2-phenylindole (DAPI) (Sigma Chemicals, U.S.A.). Tissue reactivity in groups was assessed by computerized densitom-

\section{(a)}
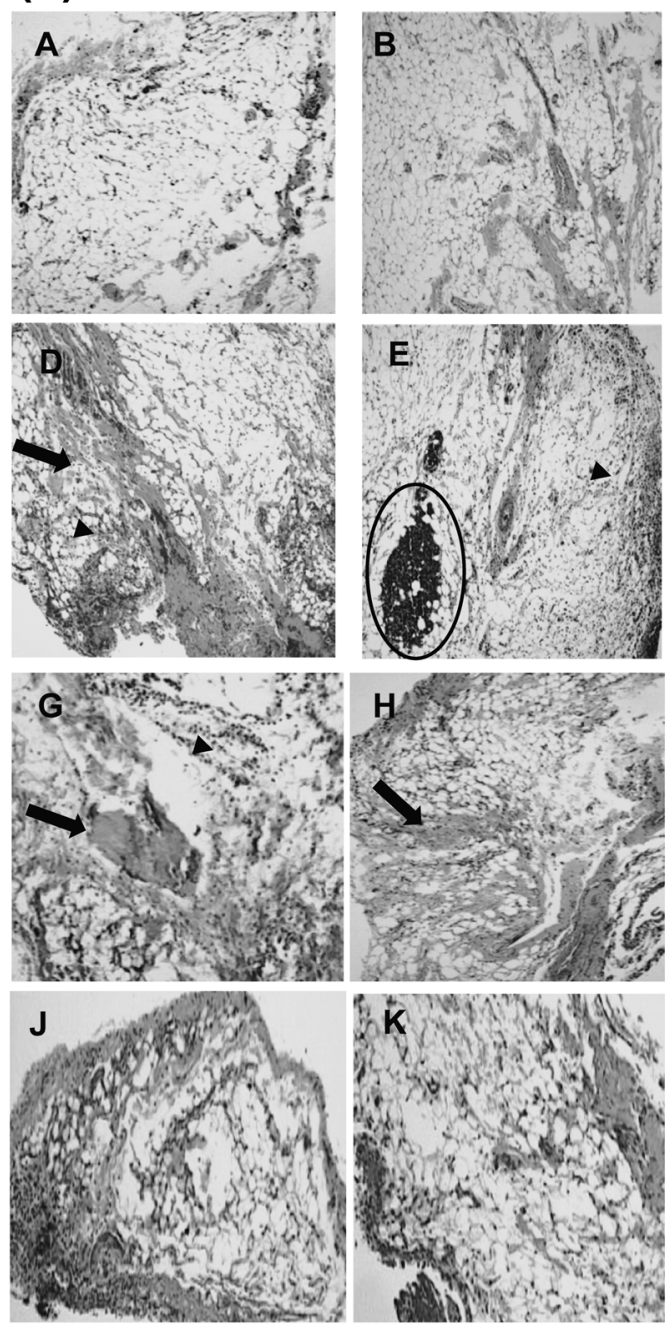
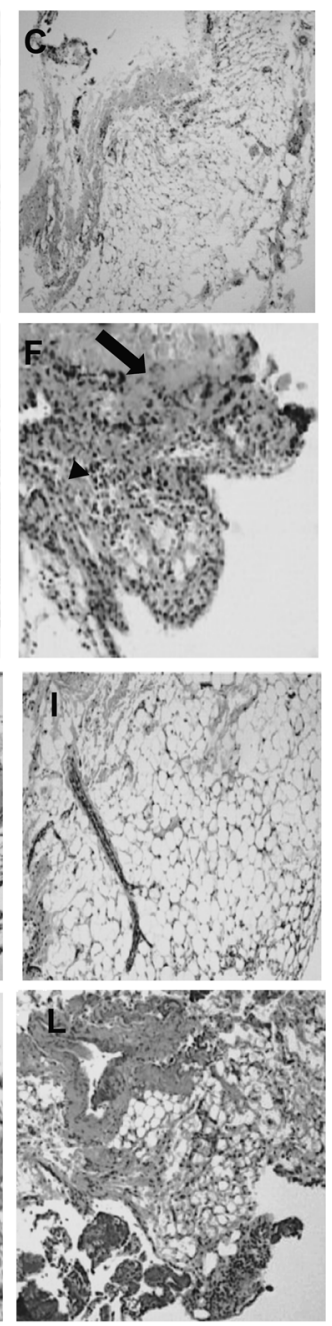

Saline

Zymosan

\section{Olmesartan}

\section{Sodium \\ Diclofenac}

(b)

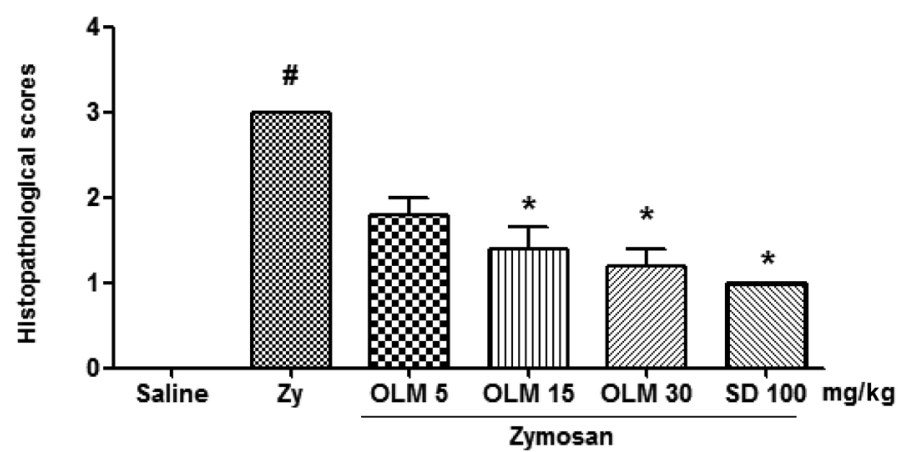

Fig. 2. (a) Histopathological Examination of the Synovial Tissue of Rats (Hematoxylin and Eosin Staining, 40×); (b) Histopathological Scores

Synovium was collected $24 \mathrm{~h}$ after zymosan administration. Synovium of animals given saline alone showed no change (A-C). Synovium of rats given zymosan alone showed fibrosis (arrow), inflammatory infiltrate (arrowhead), and granuloma (larger circles; D-F). Synovium of animals that received zymosan and were treated with $5 \mathrm{mg} /$ kg olmesartan showed fibrosis (arrow) and inflammatory infiltrate (arrowhead; G). Synovium of animals treated with OLM $15 \mathrm{mg} / \mathrm{kg}$ (H) and $30 \mathrm{mg} / \mathrm{kg}$ (I) olmesartan or diclofenac sodium $(100 \mathrm{mg} / \mathrm{kg} ; \mathrm{J}-\mathrm{L})$ showed improvement in histopathological parameters. Scores are expressed as the median \pm S.E.M. $(n=5) .{ }^{*} p<0.05 v s$. saline, ${ }^{*} p<0.05$ vs. zymosan (Kruskal-Wallis test and Dunn's multiple comparison test). 
etry analysis using digital images captured by an immunofluorescence microscope (Zeiss Imager M.2 ApoTome2). Average densitometric values were obtained using ImageJ software (http://rsb.info.nih.gov/ij/). Contrast index measurements were obtained from the selected area $\times 100 /$ total area after removing the background positioned across the regions of interest (five samples per animal). ${ }^{27}$

Analysis of Urea, Creatinine, ALT, and AST Levels Blood collection was performed by cardiac puncture following anaesthesia with thiopental. Biochemical measurements were made from serum samples, using standard LABTEST kits and a spectrophotometer. ${ }^{28)}$ The colorimetric method was used to measure urea and creatinine concentrations. ALT and AST levels were determined by kinetic assay.

Statistical Analysis Data were expressed as the mean with standard errors of the mean (S.E.M.) or the medians. ANOVA with post hoc Tukey's tests was used to compare mean values among groups. Median values were compared using the Kruskal-Wallis test and Dunn's multiple comparison test (GraphPad Prism 5.0 Software, La Jolla, CA, U.S.A.). A $p$-value $<0.05$ indicated a statistically significant difference.

\section{RESULTS}

Total Leukocyte Count and Myeloperoxidase (MPO) Activity Zy promoted joint inflammation, as evidenced by the increased number of total leukocytes in the synovial fluid and increased MPO activity in the synovia of rats compared with those in animals that received only intra-articular saline (Figs. $1 \mathrm{~A}, \mathrm{~B})$. OLM at doses of 15 and $30 \mathrm{mg} / \mathrm{kg}$ and SD improved these inflammation parameters in relation to rats that received Zy alone $(p<0.05)$. Treatment with OLM at a dose of $5 \mathrm{mg} / \mathrm{kg}$ had no significant effect (Figs. 1A, B).

Histopathology of the Synovium The synovium of untreated rats that received intra-articular injections of $\mathrm{Zy}$ showed histopathological changes, characterised by fibrosis, inflammatory infiltrate, and granuloma, with higher histopathological scores than rats that received saline (Figs. 2A, B). The synovium of rats treated with $5 \mathrm{mg} / \mathrm{kg}$ OLM showed fibrosis and inflammatory infiltrate, with no reduction in histopathological score compared with rats that received Zy alone (Figs. 2A, B). However, 15 and $30 \mathrm{mg} / \mathrm{kg}$ OLM improved inflammatory influx and eliminated fibrosis and granuloma, with a significant reduction in histopathological scores $(p<0.05$; Figs. 2A, B). SD also promoted improvement in histopathological parameters $(p<0.05$; Figs. $2 \mathrm{~A}, \mathrm{~B})$.

MDA and GSH Levels Twenty-four hours after the induction of joint inflammation with Zy, MDA levels were increased and GSH levels were reduced compared with salinetreated rats (Figs. 3A, B). OLM doses of 15 and $30 \mathrm{mg} / \mathrm{kg}$ and $\mathrm{SD}$ reduced MDA levels and restored GSH levels $(p<0.05)$, but $5 \mathrm{mg} / \mathrm{kg}$ OLM did not.

Immunohistochemical Parameters Synovia of rats with untreated Zy-induced joint inflammation showed intense immunostaining for COX-2 (Fig. 4B), TNF (Fig. 5B) and IL 17 (Fig. 5F) compared with saline-treated animals. OLM and SD reduced immunostaining for COX-2 (Figs. 4C-E), TNF (Figs. 5C, D) and IL 17 (Figs. 5G, H). Immunostaining for SOD and GSH-Px was more pronounced in the groups treated with 15 and $30 \mathrm{mg} / \mathrm{kg}$ OLM than in animals that received Zy alone (Figs. 4H, I, M, N). (a)

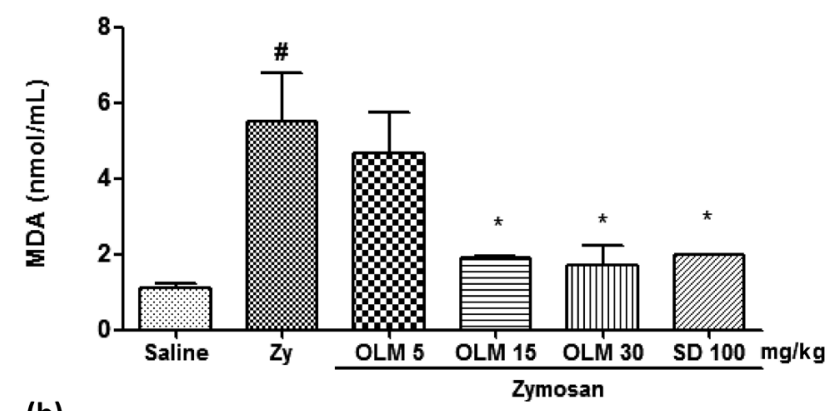

(b)

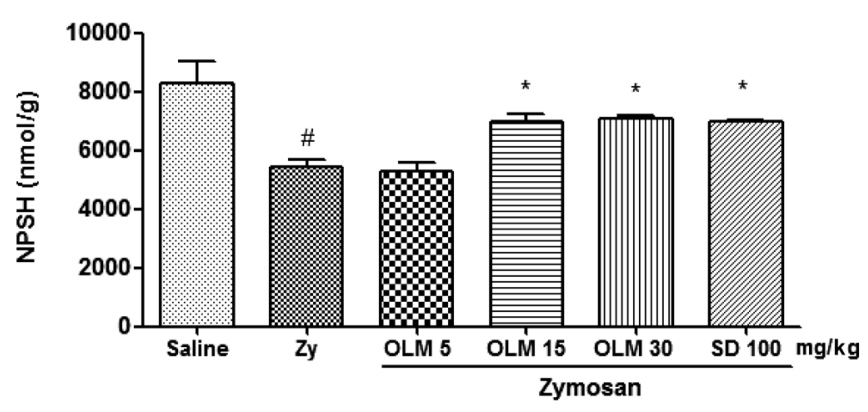

Fig. 3. Malondialdehyde (MDA) Levels (a) and Glutathione (GSH) Levels, Determined by Testing Non-protein Sulphydryl (NPSH) Groups (b)

The assays were performed on the synovia of rats collected $24 \mathrm{~h}$ after zymosan induction. Rats received intra-articular saline alone (saline), intra-articular zymosan alone (Zy), and intra-articular zymosan and olmesartan (OLM; 5, 15, or $30 \mathrm{mg}$ / $\mathrm{kg}$ ) or diclofenac sodium (SD; $100 \mathrm{mg} / \mathrm{kg}$ ). OLM and SD were administered orally $30 \mathrm{~min}$ before zymosan injection. Results are presented as the mean \pm S.E.M. $(n=5)$. ${ }^{\sharp} p<0.05$ vs. saline, ${ }^{*} p<0.05$ vs. Zy (ANOVA with Tukey's post-test).

Immunofluorescence Parameters Synovia of rats with untreated Zy-induced joint inflammation showed intense diffuse (green) in the cells for TNF (Figs. 6a, B), and IL 17 (Figs. 6a, F) compared with saline-treated animals (Figs. 6a, A, E). OLM and SD showed moderately diffuse (green) in all synovial layers for TNF (Figs. 6a, C, D) and IL 17 (Figs. 6G, $\mathrm{H})$. Densitometric analysis confirmed a significant reduction in TNF and IL 17 immunoreactivity in olmesartan and diclofenac sodium groups $(p<0.05$; Figs. $6 \mathrm{~b}, \mathrm{c})$.

Biochemical Parameters Treatment of animals with OLM $(5,15$ and $30 \mathrm{mg} / \mathrm{kg})$ caused no change in biochemical parameters, as evidenced by normal serum levels of urea, creatinine, AST, and ALT (Figs. 7a-d). SD promoted increases in the serum urea level, but not the serum creatinine, AST, or ALT level, in animals with joint inflammation $(p<0.05$; Fig. 7).

\section{DISCUSSION}

Zy induces intra-articular inflammation by binding to TLR2 expressed in synovial fibroblasts, which signals the inflammatory response. ${ }^{2)}$ In the present study, OLM had a protective effect on Zy-induced intra-articular inflammation, reducing inflammation parameters and oxidative stress.

In this study, OLM reduced the total leukocyte count, especially the number of neutrophils, as shown by the analysis of MPO activity and histopathological parameters of the synovium. Neutrophils are considered to be primary cells of the innate immune defence system that participate actively in 

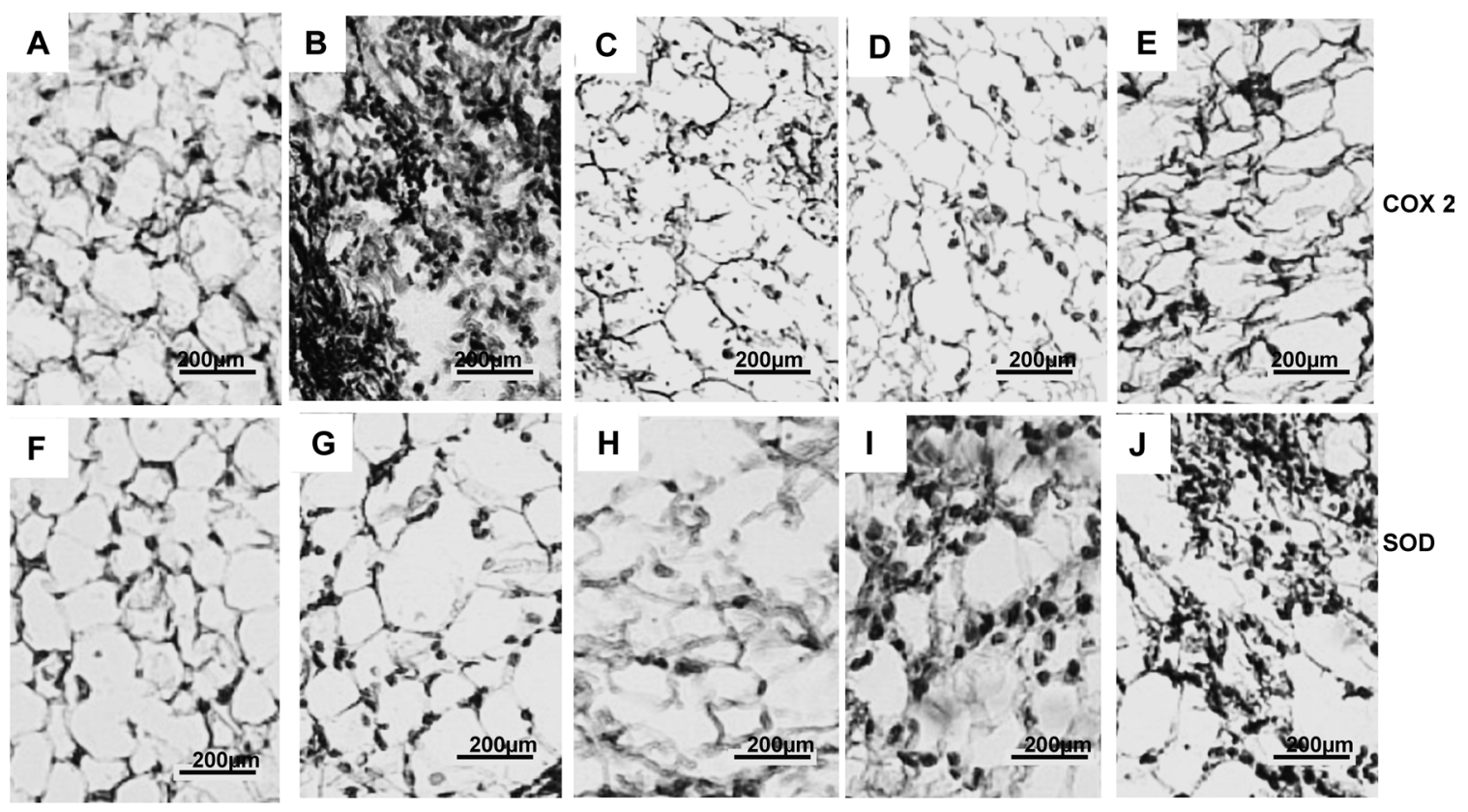

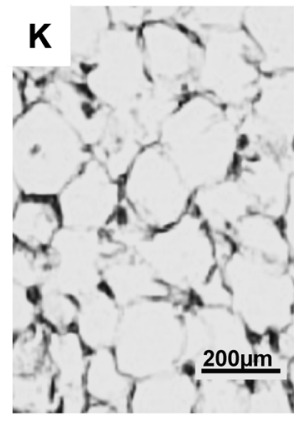

Saline

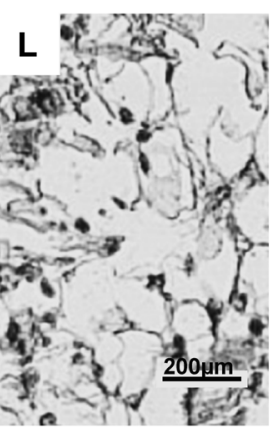

Zy

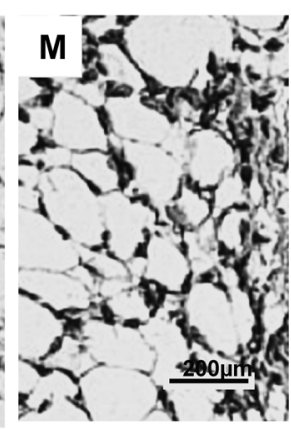

OLM 15

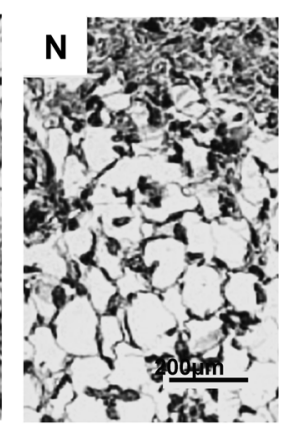

OLM 30

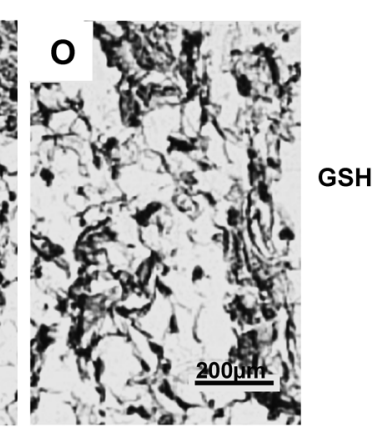

Diclofenac

Fig. 4. Representative Immunohistochemical Results for Cyclooxygenase-2 (COX-2; A-E), Superoxide Dismutase (SOD; F-J), and Glutathione Peroxidase (GSH-Px; K-O) in Synovial Tissue of Rats, Obtained 24h after Zymosan Administration

The experimental groups were saline only, zymosan only (Zy), olmesartan (OLM) 15 and 30 mg/kg, and diclofenac sodium (SD) $100 \mathrm{mg} / \mathrm{kg}$. Synovium of Zy rats showed intense immunostaining for COX-2, which was reduced by OLM and SD. Immunostaining for SOD and GSH-Px was more pronounced in OLM groups than in the Zy group.

the phagocytosis of pathogens. Recently, some authors have suggested that the formation of neutrophil extracellular traps (NETs) has the advantage of cancelling large bio-structures, such that fungi cannot be internalised by phagocytes. ${ }^{29)}$ NETs participate in the pathogenesis of autoimmune diseases; in patients with RA, NET formation stimulates synovial fibroblasts to produce pro-inflammatory mediators that participate in joint injury. ${ }^{30)}$

In this study and in other experimental models of inflammation, OLM reduced the expression of COX-2, which could result in tissue protection. ${ }^{19,31)}$ COX-2 is an enzyme induced in inflammatory processes; it is involved in the synthesis of prostaglandins and inflammatory cytokines, which promote local inflammation and tissue damage. Some authors have suggested that COX-2 expression in the synovial fibroblasts of patients with RA contributes to increased expression of inflammatory cytokines in cartilage matrix. ${ }^{32)}$ According to the results of immunohistochemistry and immunofluorescence, treatment with $30 \mathrm{mg} / \mathrm{kg}$ OLM or $100 \mathrm{mg} / \mathrm{kg}$ SD reduced TNF and IL 17 levels. OLM protected against intra-articular inflammation by down regulating immunostaining of TNF and
IL 17 in synovial tissue. These cytokines regulate the inflammatory response and activities related to joint destruction, thereby participating in RA pathogenesis. ${ }^{33,34)}$

We observed that SD, a non-steroidal anti-inflammatory agent, improved the inflammation parameters analysed. These results are similar to those observed for OLM. OLM did not promote changes in serum liver or kidney markers. SD did not cause liver abnormalities, but it did increase the serum urea level. The ability of SD to inhibit cyclooxygenases 1 and 2 in non-selective media explains this adverse effect on the renal system, ${ }^{35)}$ as well as gastrointestinal effects described previously. ${ }^{36)}$

OLM is considered to be a cardioprotective drug, due to its anti-inflammatory activity (via inhibition of the AT1 receptor of angiotensin II) and antioxidant properties. ${ }^{37,38)}$ Oxidative stress is involved in the pathogenesis of RA. ${ }^{5)}$ ROS degrade proteoglycans, activate metalloproteinases, and induce apoptosis of chondrocytes, contributing to the damage to cartilage and bone tissue. ${ }^{39)}$ We demonstrated that OLM reduced MDA levels in the synovium of rats, reflecting an improvement in lipid peroxidation, and increased levels of GSH, an impor- 

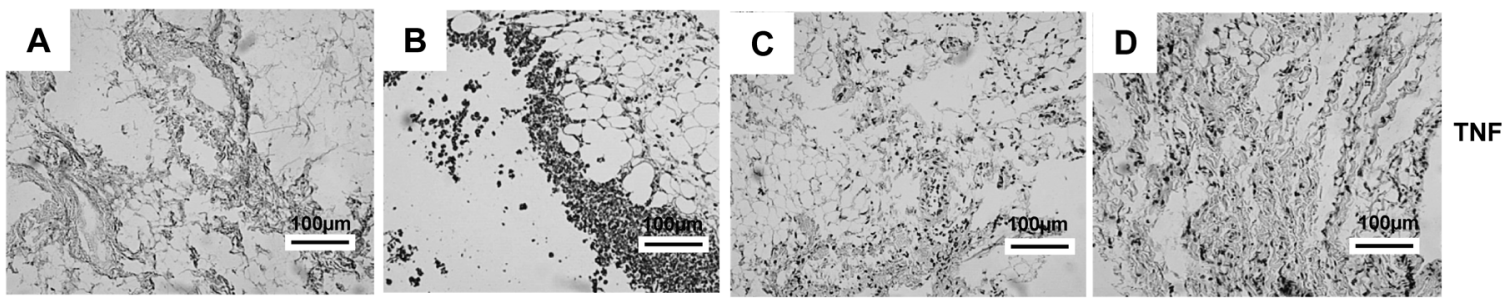

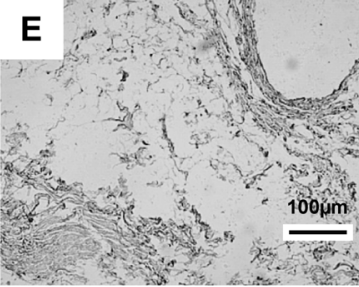

Saline

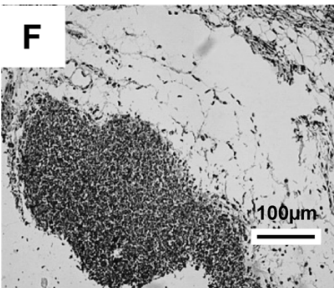

Zy

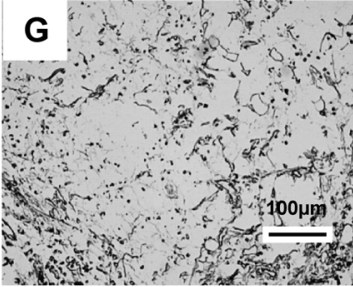

OLM 30

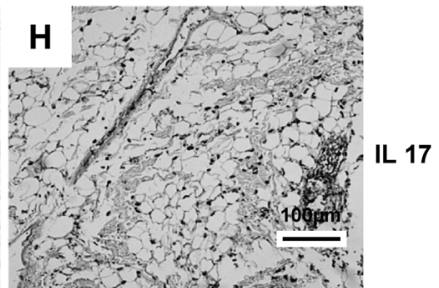

Diclofenac

Fig. 5. Representative Immunohistochemical Results for Tumour Necrosis Factor (TNF; A-D) and Interleukin 17 (IL 17 ; E-H) in Synovial Tissue of Rats, Obtained $24 \mathrm{~h}$ after Zymosan Administration

The experimental groups were saline only, zymosan only (Zy), olmesartan (OLM) $30 \mathrm{mg} / \mathrm{kg}$, and diclofenac sodium (SD) $100 \mathrm{mg} / \mathrm{kg}$. Synovium of Zy rats showed intense immunostaining for TNF and IL 17, which was reduced by OLM and SD.

(a)
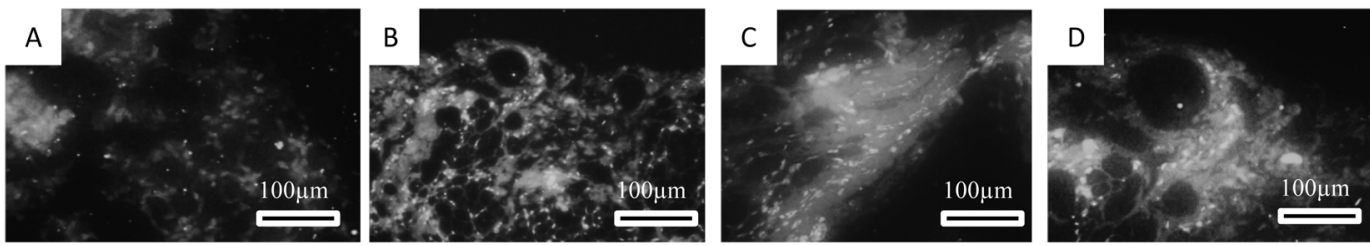

TNF

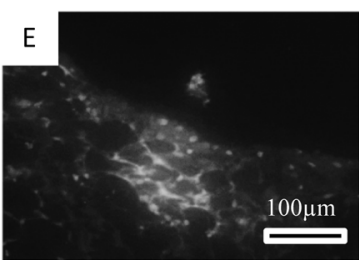

Saline

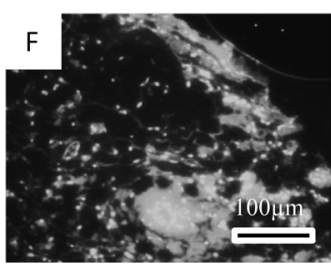

$\mathbf{Z y}$

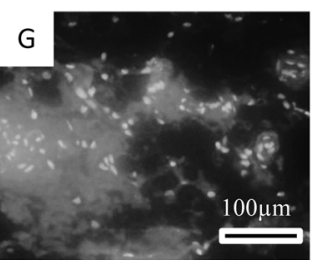

OLM 30

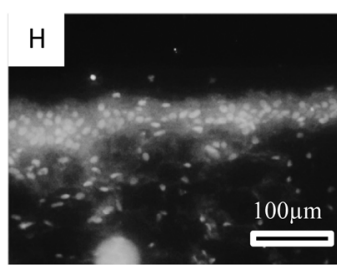

Diclofenac (b)

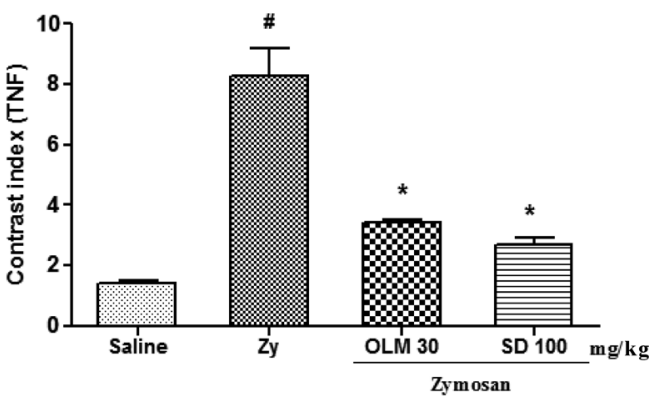

(c)

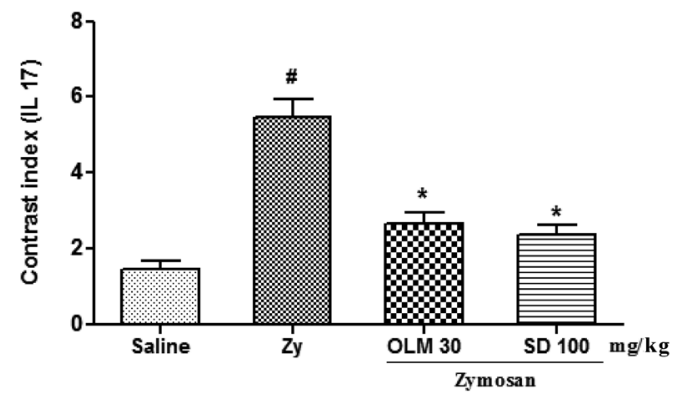

Fig. 6. Representative Immunofluorescence Results (a) and Contrast Index (b, c) for Tumour Necrosis Factor (TNF; A-D) and Interleukin 17 (IL 17; E-H) in Synovial Tissue of Rats, Obtained 24h after Zymosan Administration

The experimental groups were saline only, zymosan only (Zy), olmesartan (OLM) $30 \mathrm{mg} / \mathrm{kg}$, and diclofenac sodium (SD) $100 \mathrm{mg} / \mathrm{kg}$. Synovium of Zy rats showed intense immunoreactivity for TNF and IL 17, which was reduced by OLM and SD. ${ }^{\#} p<0.05$ vs. saline, * $p<0.05$ vs. zymosan (Kruskal-Wallis test and Dunn's multiple comparison test)

tant antioxidant, which contributed to the protective effect on intra-articular inflammation. Other authors have described the antioxidant activity of OLM. ${ }^{40,41)}$
In this study, OLM treatment increased immunostaining for antioxidant enzymes, such as SOD and GSH-Px, strengthening the evidence for this drug's protective effect on oxidative 
(a)

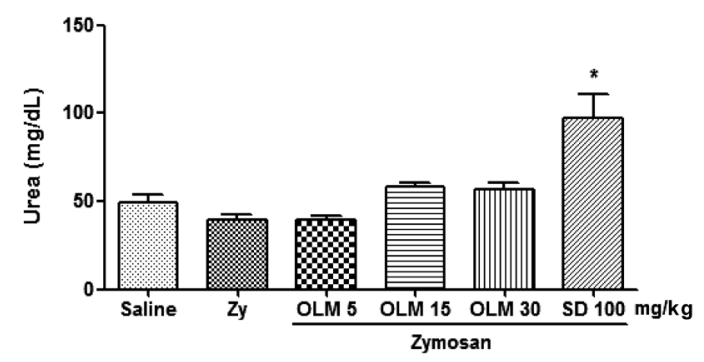

(c)

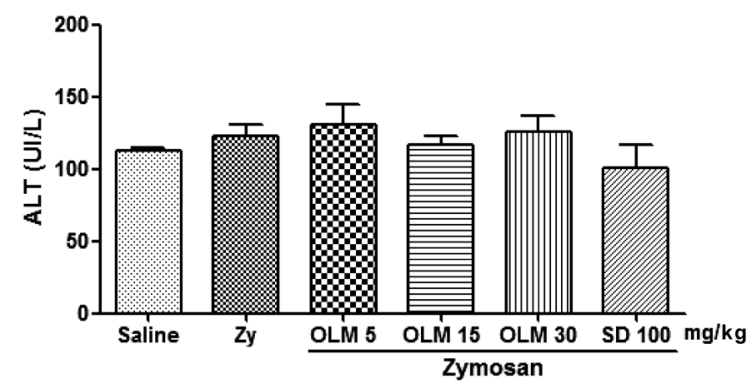

(b)

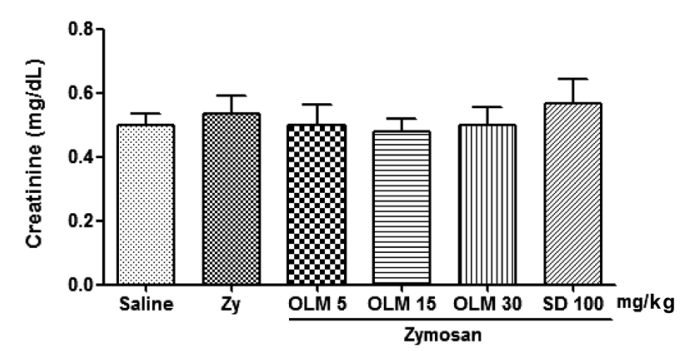

(d)

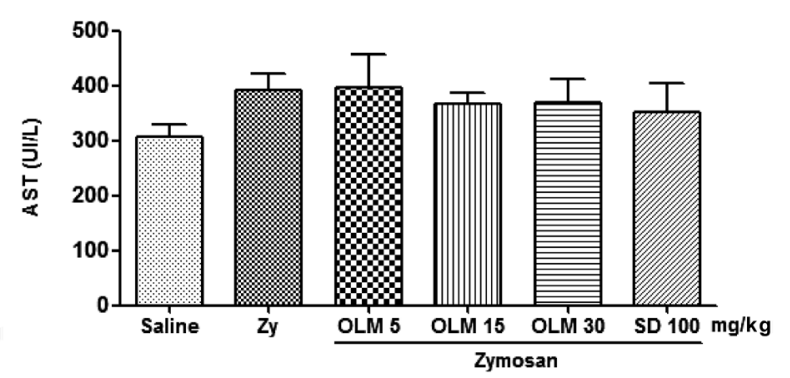

Fig. 7. Biochemical Levels of Urea (a), Creatinine (b), Alanine Aminotransferase (ALT; c), and Aspartate Aminotransferase (AST; d)

Biochemical measurements were made from serum samples of rats obtained $24 \mathrm{~h}$ after zymosan induction. Rats received intra-articular saline alone (saline), intra-articular zymosan alone (Zy), and intra-articular zymosan and olmesartan (OLM; 5, 15, or $30 \mathrm{mg} / \mathrm{kg}$ ) or diclofenac sodium (SD; 100 mg/kg). OLM and SD were administered orally $30 \mathrm{~min}$ before zymosan injection. Results are presented as the mean \pm S.E.M. $(n=5) .{ }^{\#} p<0.05 v s$. saline, ${ }^{*} p<0.05 v s$. Zy (ANOVA with Tukey's post-test).

stress. Corroborating our results, OLM increased levels of SOD in diabetic nephropathy in streptozotocin-induced diabetes in rats ${ }^{42)}$ and restored the activity of GSH-Px in daunorubicin-induced nephrotoxicity in rats. ${ }^{43)}$

Angiotensin II plays a key role in inflammation; it amplifies the inflammatory response mediated by AT1R via the activation of nuclear factor kappa B and regulates the expression of pro-inflammatory cytokines, chemokines, and adhesion molecules in living cells, contributing to the migration of inflammatory cells to sites of tissue injury. ${ }^{44,45)}$

No previous report has described the effect of OLM on Zy-induced joint inflammation. The effect of OLM in RA has been demonstrated in an experimental model of collageninduced arthritis in mice. ${ }^{46)}$ Additional studies have confirmed the anti-inflammatory activity of OLM in other models of inflammation. ${ }^{19,31,47)}$

Our results are of importance, given that RA is associated with an increased risk of CVD, and that hypertension is a prevalent risk factor for CVD in patients with RA. Thus, the use of OLM in patients with RA can bring dual benefits, due to the drug's anti-inflammatory and antihypertensive properties. Although the blocking of angiotensin II probably will not replace anti-rheumatic drugs, such as methotrexate, non-steroidal anti-inflammatory agents, and biological agents, ARB may be an antihypertensive that benefits patients with RA. We conclude that OLM had a protective effect on intra-articular inflammation and deserves further study in humans.

Conflict of Interest The authors declare no conflict of interest.

\section{REFERENCES}

1) Sweeney SE, Firestein GS. Rheumatoid arthritis: regulation of synovial inflammation. Int. J. Biochem. Cell Biol., 36, 372-378 (2004).

2) Frasnelli ME, Tarussio D, Chobaz-Peclat V, Busso N, So A. TLR2 modulates inflammation in zymosan-induced arthritis in mice. $A r$ thritis Res. Ther., 7, R370-R379 (2005).

3) Rocha FA, Aragao AG Jr, Oliveira RC, Pompeu MM, Vale MR, Ribeiro RA. Periarthritis promotes gait disturbance in zymosaninduced arthritis in rats. Inflamm. Res., 48, 485-490 (1999).

4) Jia Z, He J. Paeoniflorin ameliorates rheumatoid arthritis in rat models through oxidative stress, inflammation and cyclooxygenase 2. Experimental and Therapeutic Medicine, 11, 655-659 (2016).

5) Sahebari M, Shakeri F, Azadi HG, Arjmand MH, Ghayour-Mobarhan M, Parizadeh MR, Alamdari DH. Pro-oxidant-antioxidant balance (PAB) in rheumatoid arthritis and its relationship to disease activity. Current Rheumatology Reviews, (2015). [Epub ahead of print]

6) Cerhan JR, Saag KG, Merlino LA, Mikuls TR, Criswell LA. Antioxidant micronutrients and risk of rheumatoid arthritis in a cohort of older women. Am. J. Epidemiol., 157, 345-354 (2003).

7) Holmqvist ME, Wedren S, Jacobsson LT, Klareskog L, Nyberg F, Rantapaa-Dahlqvist S, Alfredsson L, Askling J. Rapid increase in myocardial infarction risk following diagnosis of rheumatoid arthritis amongst patients diagnosed between 1995 and 2006. J. Intern. Med., 268, 578-585 (2010).

8) Carrillo Gutiérrez OY, Pérez Sánchez AG, Medina Serriteño N, Rodríguez Orozco AR. Side effects of COX-2 selective inhibitors. Critic related with its administration in patients with rheumatoid arthritis and osteoarthritis. Rev. Alerg. Mex., 54, 116-122 (2007).

9) Bijlsma JW, Jacobs JW, Buttgereit F. Glucocorticoids in the treat- 
ment of rheumatoid arthritis. Clin. Exp. Rheumatol., 33 (Suppl. 92), S34-S36 (2015).

10) Tougaard P, Zervides KA, Skov S, Hansen AK, Pedersen AE. Biologics beyond TNF-alpha inhibitors and the effect of targeting the homologues TL1A-DR3 pathway in chronic inflammatory disorders. Immunopharmacol. Immunotoxicol., 38, 29-38 (2016).

11) Li Y, Li D, Li Y, Wu S, Jiang S, Lin T, Xia L, Shen H, Lu J. Interleukin-35 upregulates OPG and inhibits RANKL in mice with collagen-induced arthritis and fibroblast-like synoviocytes. Osteoporos. Int., 27, 1537-1546 (2016).

12) Mason RP. Optimal therapeutic strategy for treating patients with hypertension and atherosclerosis: focus on olmesartan medoxomil. Vasc. Health Risk Manag., 7, 405-416 (2011).

13) Pan Z, Feng L, Long H, Wang H, Feng J, Chen F. Effects of local pancreatic renin-angiotensin system on the microcirculation of rat with severe acute pancreatitis. The Korean Journal of Physiology \& Pharmacology, 19, 299-307 (2015).

14) Jiang Y, Jiang LL, Maimaitirexiati XM, Zhang Y, Wu L. Irbesartan attenuates TNF-alpha-induced ICAM-1, VCAM-1, and Eselectin expression through suppression of NF-kappaB pathway in HUVECs. Eur. Rev. Med. Pharmacol. Sci., 19, 3295-3302 (2015).

15) Deng W, Deng Y, Deng J, Wang DX, Zhang T. Losartan attenuated lipopolysaccharide-induced lung injury by suppression of lectinlike oxidized low-density lipoprotein receptor-1. Int. J. Clin. Exp. Pathol., 8, 15670-15676 (2015).

16) Araújo AA, Souza TO, Moura LM, Brito GA, Aragão KS, Araújo LS, Medeiros CA, Alves MS, Araújo RF Jr. Effect of telmisartan on levels of IL-1, TNF-alpha, down-regulated COX-2, MMP-2, MMP-9 and RANKL/RANK in an experimental periodontitis model. $J$. Clin. Periodontol., 40, 1104-1111 (2013).

17) Araújo AA, Varela $\mathrm{H}$, Brito GA, Medeiros CA, Araújo Lde S, do Nascimento JH, de Araújo Junior RF. Azilsartan increases levels of IL-10, down-regulates MMP-2, MMP-9, RANKL/RANK, Cathepsin $\mathrm{K}$ and up-regulates OPG in an experimental periodontitis model. PLoS ONE, 9, e96750 (2014)

18) Pacurari M, Kafoury R, Tchounwou PB, Ndebele K. The renin-angiotensin-aldosterone system in vascular inflammation and remodeling. Int. J. Inflam., 2014, 689360 (2014).

19) de Araújo RF Jr, Reinaldo MP, Brito GA, Cavalcanti Pde F, Freire MA, de Medeiros CA, de Araújo AA. Olmesartan decreased levels of IL-1beta and TNF-alpha, down-regulated MMP-2, MMP-9, COX-2, RANK/RANKL and up-regulated SOCs-1 in an intestinal mucositis model. PLoS ONE, 9, el14923 (2014).

20) Azza Z, Oudghiri M. In vivo anti-inflammatory and antiarthritic activities of aqueous extracts from Thymelaea hirsuta. Pharmacognosy Research, 7, 213-216 (2015)

21) Cardoso ML, Xavier CA, Bezerra MB, Paiva AO, Carvalho MF, Benevides NM, Rocha FA, Leite EL. Assessment of zymosaninduced leukocyte influx in a rat model using sulfated polysaccharides. Planta Med., 76, 113-119 (2010).

22) Rocha FAC, Leite AKRM, Pompeu MML, Cunha TM, Verri WA Jr, Soares FM, Castro RR, Cunha FQ. Protective effect of an extract from Ascaris suum in experimental arthritis models. Infect. Immun., 76, 2736-2745 (2008).

23) Bradley PP, Christensen RD, Rothstein G. Cellular and extracellular myeloperoxidase in pyogenic inflammation. Blood, 60, 618-622 (1982).

24) Sedlak J, Lindsay RH. Estimation of total, protein-bound, and nonprotein sulfhydryl groups in tissue with Ellman's reagent. Anal. Biochem., 25, 192-205 (1968).

25) Gérard-Monnier D, Erdelmeier I, Regnard K, Moze-Henry N, Yadan JC, Chaudiere J. Reactions of 1-methyl-2-phenylindole with malondialdehyde and 4-hydroxyalkenals. Analytical applications to a colorimetric assay of lipid peroxidation. Chem. Res. Toxicol., 11, $1176-1183$ (1998)

26) Hsu SM, Raine L. Protein A, avidin, and biotin in immunohisto- chemistry. J. Histochem. Cytochem., 29, 1349-1353 (1981).

27) Araújo Junior RF, Garcia VB, Leitão RF, Brito GA, Miguel Ede C, Guedes PM, de Araújo AA. Carvedilol improves inflammatory response, oxidative stress and fibrosis in the alcohol-induced liver injury in rats by regulating kuppfer cells and hepatic stellate cells. PLOS ONE, 11, e0148868 (2016).

28) Medeiros CA, Leitao RF, Macedo RN, Barboza DR, Gomes AS, Nogueira NA, Alencar NM, Ribeiro RA, Brito GA. Effect of atorvastatin on 5-fluorouracil-induced experimental oral mucositis. Cancer Chemother. Pharmacol., 67, 1085-1100 (2011).

29) Pruchniak MP, Arazna M, Demkow U. Life of neutrophil: from stem cell to neutrophil extracellular trap. Respir. Physiol. Neurobiol., 187, 68-73 (2013).

30) Pratesi F, Dioni I, Tommasi C, Alcaro MC, Paolini I, Barbetti F, Boscaro F, Panza F, Puxeddu I, Rovero P, Migliorini P. Antibodies from patients with rheumatoid arthritis target citrullinated histone 4 contained in neutrophils extracellular traps. Ann. Rheum. Dis., 73, 1414-1422 (2014).

31) Araujo AA, Lopes de Souza G, Souza TO, de Castro Brito GA, Saboia Aragao K, Xavier de Medeiros CA, Lourenco Y, do Socorro Costa Feitosa Alves M, Fernandes de Araujo R Jr. Olmesartan decreases IL-1beta and TNF-alpha levels; downregulates MMP-2, MMP-9, COX-2, and RANKL; and upregulates OPG in experimental periodontitis. Naunyn Schmiedebergs Arch. Pharmacol., 386, 875-884 (2013).

32) Huang QC, Huang RY. The cyclooxygenase-2/thromboxane A2 pathway: a bridge from rheumatoid arthritis to lung cancer? Cancer Lett., 354, 28-32 (2014).

33) Ryu S, Lee JH, Kim SI. IL-17 increased the production of vascular endothelial growth factor in rheumatoid arthritis synoviocytes. Clin. Rheumatol., 25, 16-20 (2006).

34) Hata H, Sakaguchi N, Yoshitomi H, Iwakura Y, Sekikawa K, Azuma Y, Kanai C, Moriizumi E, Nomura T, Nakamura T, Sakaguchi S. Distinct contribution of IL-6, TNF-alpha, IL-1, and IL-10 to T cell-mediated spontaneous autoimmune arthritis in mice. J. Clin. Invest., 114, 582-588 (2004).

35) Besen A, Kose F, Paydas S, Gonlusen G, Inal T, Dogan A, Kibar M, Balal M. The effects of the nonsteroidal anti-inflammatory drug diclofenac sodium on the rat kidney, and alteration by furosemide. Int. Urol. Nephrol., 41, 919-926 (2009).

36) Moore RA, Derry S, McQuay HJ. Cyclo-oxygenase-2 selective inhibitors and nonsteroidal anti-inflammatory drugs: balancing gastrointestinal and cardiovascular risk. BMC Musculoskelet. Disord., 8, 73 (2007).

37) $\overline{\mathrm{Lu} \mathrm{X}, \mathrm{Bi} \mathrm{YW}}$, Chen KB, Wang HY. Protective effect of olmesartan against cardiac ischemia/reperfusion injury in spontaneously hypertensive rats. Experimental and Therapeutic Medicine, 9, 2081-2087 (2015).

38) Calò LA, Maso LD, Caielli P, Pagnin E, Fusaro M, Davis PA, Pessina AC. Effect of olmesartan on oxidative stress in hypertensive patients: mechanistic support to clinical trials derived evidence. Blood Press., 20, 376-382 (2011).

39) Manunta AF, Zedde P, Cudoni S, Caggiari G, Pintus G. Early joint degeneration and antagonism between growth factors and reactive oxygen species. Is non-surgical management possible? Joints, 3, 123-128 (2015)

40) Güler A, Sahin MA, Ucak A, Onan B, Inan K, Oztas E, Arslan S, Uysal B, Demirkilic U, Tatar H. Protective effects of angiotensin II type-1 receptor blockade with olmesartan on spinal cord ischemiareperfusion injury: an experimental study on rats. Ann. Vasc. Surg., 24, 801-808 (2010)

41) Chuang YC, Wu MS, Su YK, Fang KM. Effects of olmesartan on arterial stiffness in rats with chronic renal failure. Cardiovasc. Diabetol., 11, 66 (2012).

42) Si X, Li P, Zhang Y, Zhang Y, Lv W, Qi D. Renoprotective effects of olmesartan medoxomil on diabetic nephropathy in streptozoto- 
cin-induced diabetes in rats. Biomedical Reports, 2, 24-28 (2014).

43) Gounder VK, Arumugam S, Arozal W, Thandavarayan RA, Pitchaimani V, Harima M, Suzuki K, Nomoto M, Watanabe K. Olmesartan protects against oxidative stress possibly through the Nrf2 signaling pathway and inhibits inflammation in daunorubicininduced nephrotoxicity in rats. Int. Immunopharmacol., 18, 282-289 (2014).

44) Chan YC, Leung PS. Angiotensin II type 1 receptor-dependent nuclear factor-kappaB activation-mediated proinflammatory actions in a rat model of obstructive acute pancreatitis. J. Pharmacol. Exp. Ther., 323, 10-18 (2007)

45) Dandona P, Dhindsa S, Ghanim H, Chaudhuri A. Angiotensin II and inflammation: the effect of angiotensin-converting enzyme inhibition and angiotensin II receptor blockade. J. Hum. Hypertens., 21, 20-27 (2007)

46) Sagawa K, Nagatani K, Komagata Y, Yamamoto K. Angiotensin receptor blockers suppress antigen-specific $\mathrm{T}$ cell responses and ameliorate collagen-induced arthritis in mice. Arthritis Rheum., 52, 1920-1928 (2005).

47) Nagib MM, Tadros MG, ElSayed MI, Khalifa AE. Anti-inflammatory and anti-oxidant activities of olmesartan medoxomil ameliorate experimental colitis in rats. Toxicol. Appl. Pharmacol., 271, 106-113 (2013). 